\title{
Connecting conduit-free and bypass circuit-less mini LVADs eliminate the most likely sites of thrombosis
}

\author{
Kun-Xi Qian ${ }^{1}$ \\ ${ }^{1}$ Institute of BME, Jiangsu University, Zhenjiang, China. \\ Email: kxqian@263.net
}

Received 9 April 2009; revised 5 May 2009; accepted 7 May 2009. most favorite to be formed in these conduits and circuit, especially near their connecting sites; and the bypass flow will disturb the flow patterns in ventricle as well as in aorta which increases the danger of thrombosis.
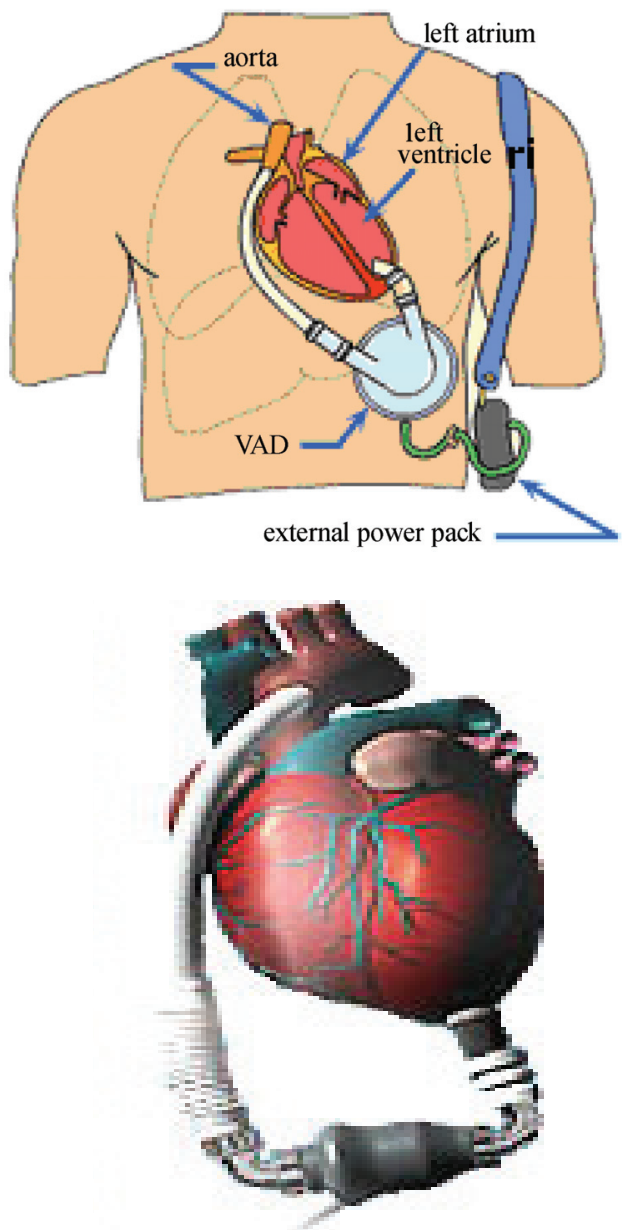

Figure 1. Diaphragm type (upper) and rotary type (lower) of LVADs on the patients. Their inlet and outlet connecting conduits together with the device form a bypass circuit, resulting in several most likely sites of thrombosis, bringing about physiological disturbance to natural circulation as well as additional need for anatomic occupation in the chest of the patients. 
Furthermore, this bypass circuit including connecting conduits and the LVAD itself needs additional anatomic occupation and that is hardly possible to be implanted in the patients' chest. Therefore, most available LVADs should remain outside of the body of the patients, increasing the danger of infection.

Because the difficulties in design and manufacture of mini LVADs, other investigators have long since not succeeded in developing a fully implantable intra- ventricular pump without inlet and outlet conduits and without bypass circuit $[1,2,3]$ but their idea leads the author to develop a trans-apical and cross-valvular intra-ventricular axial pump and an aortic valvo-pump finally. These devices generally solved the problems of thrombus formation outside the pump in left ventricular assist.

\section{TRANS-APICAL AND CROSS- VALVULAR INTRA-VENTRICULAR AXIAL PUMP}

The device has a motor and a pump entirely contained within one cannula (Figure 2(a)). The motor has a stator coil with iron core and a rotor with 4-pole magnet; the pump has an impeller and an outflow guide vane (Figure 2(b)). The motor part has $60 \mathrm{~mm}$ length and $13 \mathrm{~mm}$ diameter; the pump part has $55 \mathrm{~mm}$ length and $11 \mathrm{~mm}$ diameter. Therefore, the total length of the device is 115 $\mathrm{mm}$. The total weight of the device is $53 \mathrm{~g}$. The motor uses rolling bearing with 8 needles on each side of the rotor magnets. A special purge system is devised for the infusion of saline mixed with heparin through bearing to the pump inlet. Thus the bearing needles work in saline and neither mechanical wear nor thrombus formation along the bearing will occur.

In its hemo-dynamic testing, the pump can produce a flow of $10 \mathrm{l} / \mathrm{min}$ with $80 \mathrm{mmHg}$ pressure increase, at 15,000 rpm rotating speed of the pump (Figure 2(c)). At zero flow rate corresponding to diastolic period of the heart, the pump can maintain aortic blood pressure over $80 \mathrm{mmHg}$ at $12500 \mathrm{rpm}$ rotating speed.

This novel pump can be inserted into the ventricle via apex and extended into the aorta across the aortic valve. Thus it needs no inlet and outlet connecting conduits and no bypass circuits, delivers blood directly from ventricle to aorta without disturbance to natural circulation, requires no anatomic occupation in the chest, and can be quickly inserted in an emergency and easily removed after recovery of natural heart. Therefore, this device is suitable for recovery therapy.

\section{AORTIC VALVO-PUMP}

Aortic valvo-pump (Figure 3(a)) delivers blood flow also directly from ventricle to aorta and needs no inlet and outlet connecting tubes too. Similarly, it needs no bypass circuit, will not disturb the natural circulation, and needs no additional anatomic occupation. The device can be sewed onto the aortic valve annulus via sewing ring (Figure 3(b)). It has a rotor and a stator, the

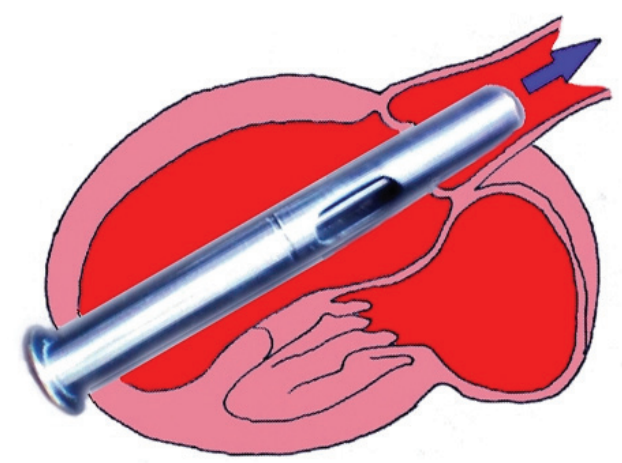

(a)

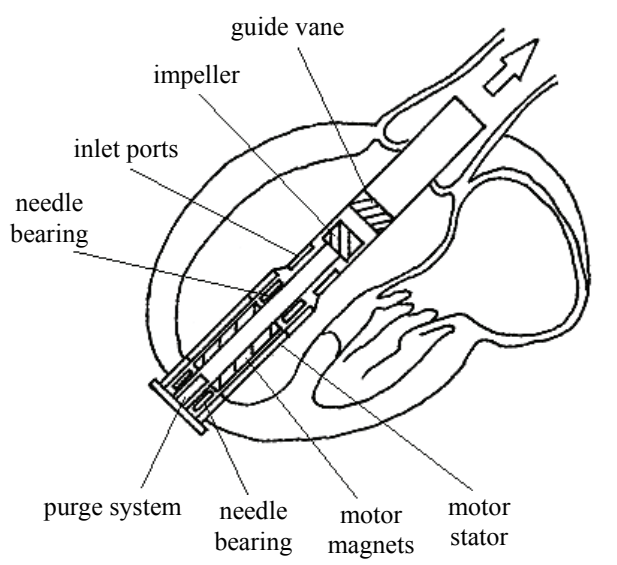

(b)

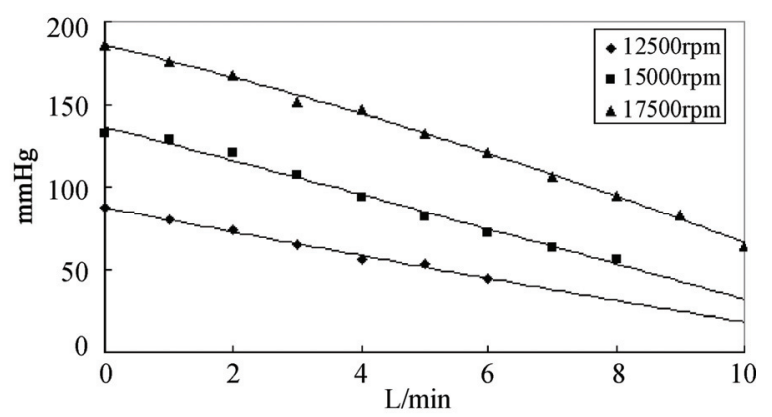

(c)

Figure 2. Trans-apical and cross-valvular intra-ventricular axial pump (above) delivers the blood flow directly from ventricle to aorta, without need for inlet and outlet tubes, without bypass circuit, without physiologic disturbance to natural circulation, without additional need for anatomic occupation. Specially designed purge system and needle bearing (middle) enable the motor and the pump to work in saline and hereby to achieve excellent antithrombogenecity and durability. Bench testing demonstrated the pump can deliver $101 / \mathrm{min}$ flow against $80 \mathrm{mmHg}$ pressure at $15000 \mathrm{rpm}$ rotating speed (below). 


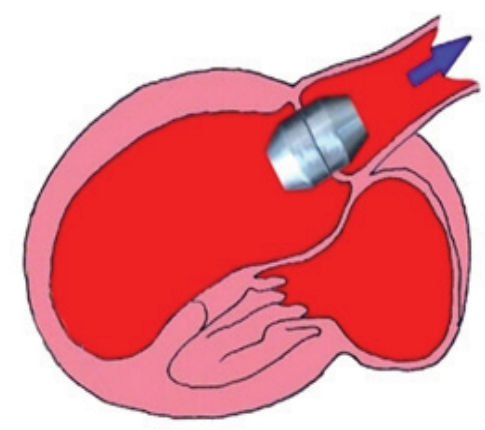

(a)

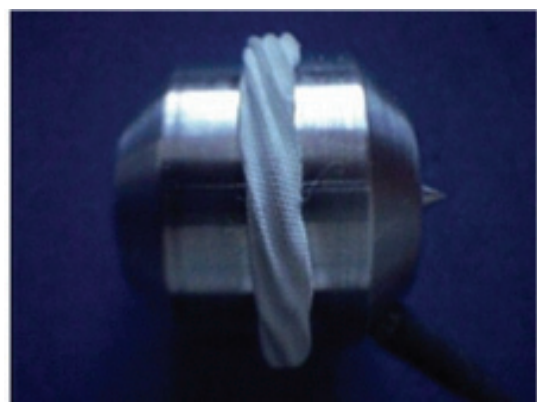

(b)

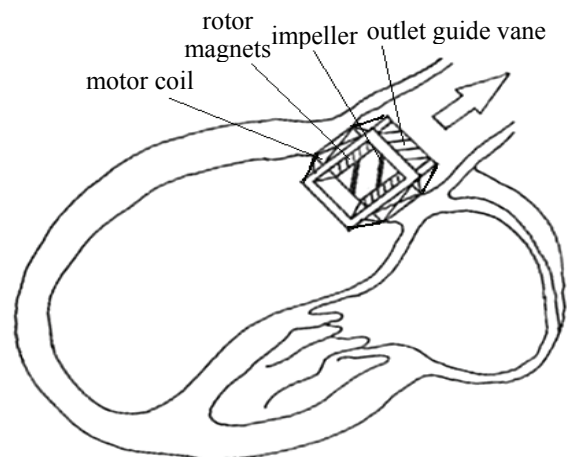

(c)

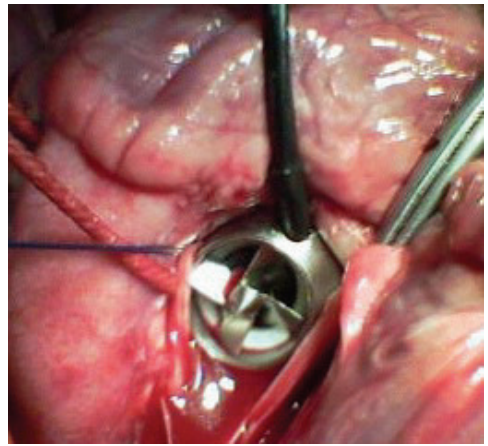

(d)

Figure 3. Aortic valvo-pump (a) and its first animal experiment (d). The pump can be sewed onto the aortic valve annulus by sewing ring (b) without harm to adjacent tissues, cells and organ functions, delivers blood directly from ventricle to aorta, needs no inlet and outlet tubes, needs no additional anatomic occupation, has no physiologic disturbance to natural circulation. The bearing-less design with enclosed impeller (c) ensures the rotor suspended in blood flow and thus the mechanical wear will not occur in the device. former has an enclosed impeller and driven magnets, the latter consists of a motor coil with iron core and an outflow guide vane (Figure 3(c)). This bearing-less design with enclosed impeller has advantages of no existence of stasis and dead-water area in the pump, that has been long since acknowledged to being favorite sites of coagulation and thrombus formation. The rotor has a gap of $0,25 \mathrm{~mm}$ in radius with the stator, a back-flow of about $0,81 / \mathrm{min}$ flows from outlet higher pressure area to inlet lower pressure area via this gap; thereby, wash-out of the gap and hydraulic suspension of the rotor will be achieved during pumping (Figure 3(c)). The devices' outer diameters are $21 \mathrm{~mm}, 23 \mathrm{~mm}$ and $25 \mathrm{~mm}$ respectively, weighing 27 gram(inc. rotor $5 \mathrm{~g}$ ), 31 gram (inc. rotor $7 \mathrm{~g}$ ) and $40 \mathrm{gram}$ (inc. rotor $11 \mathrm{~g}$ ) separately. The laboratory testing demonstrated that the rotating speed for maintaining a diastolic pressure of $80 \mathrm{mmHg}$ at zero flow rate should be $17500 \mathrm{rpm}, 15000 \mathrm{rpm}$ and $12500 \mathrm{rpm}$ correspondingly, the largest flow at these same speeds will be $51 / \mathrm{min}, 71 / \mathrm{min}$ and $101 / \mathrm{min}$ relatively, with ca. $50 \mathrm{mmHg}$ pressure increase. Therefore, these three pumps may meet with the hemo-dynamic requirements of $40-60 \mathrm{~kg}, 60-80 \mathrm{~kg}$ and $80-100 \mathrm{~kg}$ body weight patients. The first in vivo trial exhibited that the $25 \mathrm{~mm}$ valvopump could be sewed onto the aortic valve annulus of one $80 \mathrm{~kg}$ body weight pig easily (Figure 3(d)), without harm to adjacent cells, tissues and organ functions. A mentioned above, the devices have no inlet and outlet connecting tubes and no bypass circuit, occupy no additional anatomic space and deliver the blood directly from ventricle to the aorta, having thus less physiologic disturbance to the natural circulation.

\section{DISCUSSION}

The idea of trans-apical and cross-valvular intra- ventricular axial pump and aortic valvo-pump existed before. The contribution of this paper is minimizing the motor dimension and weight without decreasing its output torque so as to maintain the efficient hemo-dynamic capacity of the pump. This is a result of the author's profound investigation on motor miniaturization [4].

In this paper the author deals with the thrombosis problem outside the pump in left ventricular assist pump. For the same problem inside the pump, the author has much more extensive studies before $[5,6,7]$.

These new devices have to be further investigated in animal experiments. The author searches cooperation with other investigators all over the world.

\section{REFERENCES}

[1] K. Yamazaki, M. Umezu, H. Koyanagi, M. Kitamura, K. Eishi, A. Kawai, O. Tagusari, H. Niinami, T. Akimoto, C. Nojiri, K. Tsuchiya, T. Mori, H. Iiyama, and M. Endo, (1992) A miniature intraventricular axial flow blood pump 
that is introduced through the left ventricular apex, Trans ASAIO, 38, 679-683.

[2] H. Mitamura, H. Nakamura, E. Okamoto, R. Yozu, S. Kawada, and D. W. Kim, (1999) Development of the valvo pump: An axial flow pump implanted at the heart valve position, Artificial Organs, 23(6), 566.

[3] G. Li, H. Zhao, X. Zhu, and B. Ren, (2002) Preliminary in vivo study of an intra-aortic impeller pump driven by an extracorporeal whirling magnet, Artif Organs, 26(10), 890.

[4] K. X. Qian, H. Y. Yuan, W. M. Ru, and P. Zeng, (2002) Experimental method to reveal the effect of rotor magnet size and air-gap on artificial heart driving motor torque and efficiency, J Med. Eng. Tech. 26(5), 199-201.

[5] K. X. Qian, (2001) Axial reciprocation of rotating impeller: a new concept of antithrombogenecity in centrifugal pump, Journal of Medical Engineering \& Technology, 25(1), 25-27.

[6] K. X. Qian, P. Zeng, W. M. Ru, H. Y. Yuan, (2003) A novel permanent maglev impeller TAH: Most requirements on blood pumps have been satisfied, Journal of Biomaterials Applications, 18(1), 53-61.

[7] K. X. Qian, (1990) Haemodynamic approach to reducing thrombosis and haemolysis in an impeller pump, J Biomed Eng., 12(6), 533-535. 